\title{
Mono- and co-cultures of bronchial and alveolar epithelial cells respond differently to pro-inflammatory stimuli and their modulation by salbutamol and budesonide
}

Mehra Haghi, ${ }^{1,2,3}$ Marius Hittinger, ${ }^{1}$ Qingxiang Zeng, ${ }^{4}$ Brian Oliver, ${ }^{3,5}$ Daniela Traini, ${ }^{3}$ Paul M. Young, ${ }^{3}$ Hanno Huwer, ${ }^{6}$ Nicole Schneider-Daum, ${ }^{1 *}$ and Claus-Michael Lehr ${ }^{1}$

${ }^{1}$ Drug Delivery, Helmholtz Institute for Pharmaceutical Research Saarland (HIPS), Helmholtz Centre for Infection Research (HZI), 66123 Saarbrücken, Germany

${ }^{2}$ School of Pharmacy, Graduate School of Health, The University of Technology, Sydney, NSW 2007, Australia

${ }^{3}$ Woolcock Institute for Medical Research and Discipline of Pharmacology, Sydney Medical School, University of Sydney, NSW 2006, Australia

${ }^{4}$ Otorhinolaryngology Hospital, The First Affiliated Hospital of Sun Yat-sen University, Guangzhou 510080, China

${ }^{5}$ School of Medical \& Molecular Biosciences, University of Technology Sydney, Sydney, NSW 2007, Australia

${ }^{6}$ Department of Cardiothoracic Surgery, Voelklingen Heart Centre, 66333 Völklingen, Germany

*Corresponding author: Nicole Schneider-Daum, Address: Drug Delivery, Helmholtz Institute for Pharmaceutical Research Saarland (HIPS), Helmholtz Centre for Infection Research (HZI), Saarbrücken, Germany, Telephone + 49-302-2814, Fax +49-302-4677, email Nicole.Daum@helmholtz-hzi.de 
Keywords

alveolar macrophages, airway smooth muscle cells, epithelial transport, inflammation, cytokine

\section{Table of content graphic}

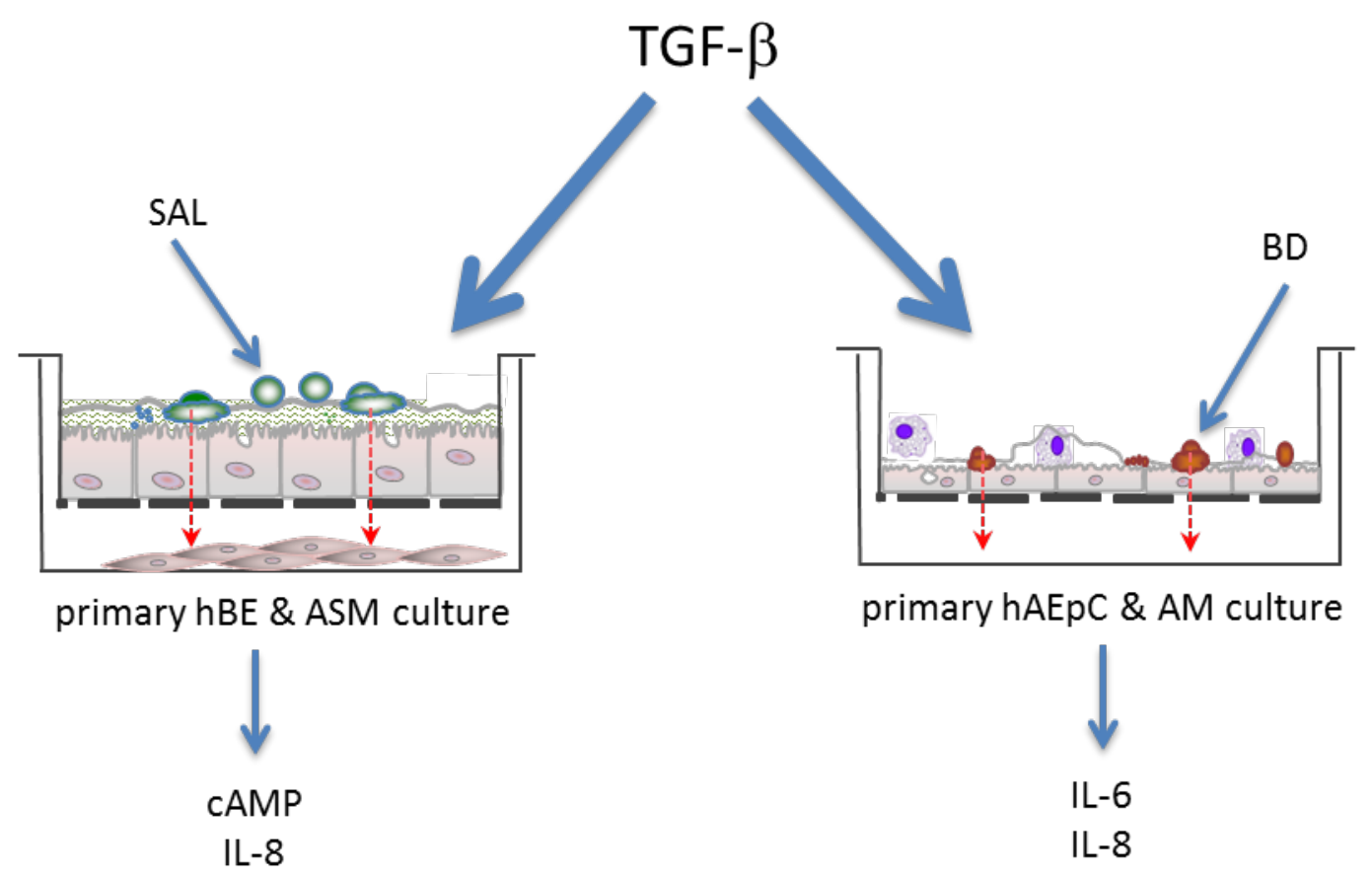




\section{Abstract}

The aim of this study was to investigate the changes in transport and effectiveness of salbutamol sulphate (SAL) and budesonide (BD) following stimulation with transforming growth factor- $\beta$ (TGF- $\beta$ ) in mono- and co-culture models of bronchial and alveolar epithelium.

Primary bronchial and alveolar epithelial cells, grown at air interface on filters, either as monocultures or in co- culture with airway smooth muscle cells or alveolar macrophages, respectively, were stimulated with TGF- $\beta$. The biological response was modulated by depositing aerosolised SAL and BD on bronchial and alveolar models, respectively. Barrier integrity, permeability to fluorescein-Na, transport of the deposited drug and the pharmacological response to SAL (cAMP and IL-8 levels) or BD (IL-6 and -8 levels) were measured.

While stimulation with TGF- $\beta$ did not have any significant effect on the transepithelial electrical resistance and permeability to fluorescein-Na in mono- and co- culture models, transport of SAL and BD were affected in cultures from some of the patients (6 out of 10 for bronchial and 2 out of 4 for alveolar cells). The bronchial co-culture showed a better responsiveness to SAL in terms of cAMP release than the monoculture. In contrast, the difference between alveolar mono- and co-cultures to TGF- $\beta$ mediated interleukin release and its modulation by BD was less pronounced.

Our data point to intrinsic differences in the transport of, and responsiveness to, SAL and BD when epithelial cell cultures originate from different patients. Moreover, if the biological responses (e.g. IL-8, cAMP) involve communication between different cell types, co-culture models are more relevant to measure such effects than monocultures. 


\section{Introduction}

Chronic obstructive pulmonary disease (COPD) and asthma are major and increasing health problems worldwide [1, 2] causing considerable morbidity and mortality. Despite the fact that asthma and chronic obstructive pulmonary disease (COPD) are both featured by airway remodelling and chronic inflammation of the airways, there are differences in the site of inflammation as well as the inflammatory cells involved, suggesting different patterns of mediators in each disease [3].

$\beta_{2}$-agonists and inhaled corticosteroids improve clinical outcome of COPD and asthma. The Global Initiative for Asthma (GINA) reports salbutamol sulphate (SAL) to be the most widely used short- and quick-acting airway bronchodilator, while budesonide (BD) is mentioned as one of the most effective anti-inflammatory inhaled corticosteroids (ICS) that markedly reduces the frequency and severity of asthma symptoms. Global Strategy for Diagnosis, Management, and Prevention of COPD (GOLD) reports the efficacy and safety of BD in the management of COPD $[1,2]$. SAL binds to $\beta_{2}$-adrenergic receptors on bronchial smooth muscle cells that consequentially result in the activation of adenylyl cyclase, and subsequent cyclic adenosine monophosphate (cAMP)-mediated smooth muscle relaxation [4]. However, BD exerts its antiinflammatory effect by suppressing the inflammatory gene transcription in asthma, COPD and other inflammatory diseases [5].

Remodelling, a well-known feature of severe asthma and COPD, involves many inflammatory and structural cells that release multiple mediators. The transforming growth factor- $\beta$ (TGF- $\beta$ ) regulates cellular functions such as proliferation, apoptosis, differentiation, and migration. The regulatory role of TGF- $\beta$ in airway remodelling has been shown in several studies [6, 7]. In 
addition, TGF- $\beta$ acts as a pro-fibrotic and immune-modulatory cytokine, is increased in patients with severe diseases such as COPD and has been shown to play a pivotal role in airway structural changes [8]. Macrophages, as well as airway and alveolar epithelial cells, may be an important source of inflammatory mediators, including TGF- $\beta$, which in turn stimulates the synthesis of extra cellular matrix components leading to local fibrosis $[9,10]$. It has also been shown that TGF- $\beta$ down regulates the $\beta_{2}$-adrenergic receptors, attenuating the effects of therapeutically applied $\beta_{2}$-adrenergic agonists $[10,11]$.

In patients with COPD and asthma, the preferable route of administration for the $\beta_{2}$-agonists and steroids is via inhalation. When drug microparticles are aerosolised and inhaled, they deposit at different regions of the airways on epithelial cells. In order for these drugs to be therapeutically effective they then need to be transported through the bronchial epithelial cell layer to reach the underlying mesenchymal and inflammatory cells. In clinical practice it is common to progressively increase the dose of steroids in an attempt to control the disease. However, in steroid insensitive patients, this often results in long-term treatment with high doses of steroids, causing significant side effects [12, 13]. Hence, understanding the reason behind insensitivity to corticosteroids is crucial. There are many plausible reasons why steroids are ineffective in COPD and severe asthma. Hence, the majority of research has focused either on elucidating the molecular mechanisms behind those diseases or on measuring the therapeutic outcome in clinical settings [14-19]. The use of advanced in vitro models based on human cells and tissues for investigating the mechanisms, safety and efficacy of therapeutic modalities is rather new, but may significantly facilitate the translation of new, safe, and better medicines into the clinic. The recently published review of Hittinger et al. focuses on this topic and offers a broad overview of preclinical in vitro models [20]. 
In the last decade, transport of drug molecules across primary airway epithelial cells and respiratory epithelial cell lines have been used to determine the fate of drug microparticles after deposition on airway epithelia [20-22]. A previous study by the author has reported that the transport of steroids through lung epithelial cell lines is markedly ineffective [23], which led to the hypothesis that epithelial remodelling in COPD and asthma may contribute in exacerbations and unresponsiveness to standard $\beta_{2}$-agonist and corticosteroid therapy, due to changes in epithelial cell permeability and in cytokine secretion.

The aim of this study was to investigate mono- and co-culture models representing the characteristics of the bronchial and alveolar epithelium following stimulation with TGF- $\beta$ to allow monitoring relevant pathological changes upon pro-inflammatory stimuli, such as TGF$\beta$, as well as their modulation by SAL and BD. 


\section{Experimental Section}

\section{Human bronchial epithelial (hBE) and airway smooth muscle (ASM) cell isolation and culture}

\section{$\mathrm{hBE}$ and $\mathrm{ASM}$ cell isolation}

Bronchial epithelial and ASM cells were isolated from human bronchial airways obtained from patients undergoing lung resection or transplantation. hBE cells were maintained and expanded separately in Ham's F-12 with growth supplements, while ASM cells were maintained in DMEM supplemented with 10\% FBS as previously described [24]. The Ethics Review Committee of the South West Sydney Area Health Service, Royal Prince Alfred Hospital and the University of Sydney Human Research Ethics Committee provided approval for the experiments.

\section{Air- interface culture of hBE cells}

Bronchial epithelial cells at passage 2 or 3 were seeded onto Transwell ${ }^{\circledR}$ polyester inserts $(0.33$

$\mathrm{cm}^{2}, 0.4 \mu \mathrm{m}$ pore size) (Corning Costar, Lowell, MA, USA) (density: $3 \times 10^{5}$ cells per insert). The growth medium in the apical chamber was removed when the cells were confluent and the bronchial epithelial growth medium (BEGM) (Lonza, Melbourne, Australia) in the basal chamber was replaced by bronchial epithelial differentiation medium (BEDM) (Lonza, Melbourne, Australia). hBE cells were allowed to differentiate under air-interface over 21-28 days. Differentiation was confirmed through mucus secretion and cilia formation and beating (see supplementary materials). 


\section{Co-culture of hBE cells and ASM cells}

21 days following of seeding of hBE cells on Transwells ${ }^{\circledR}$, passages 3-7 of the ASM cells derived from the same patient were seeded in 24-well culture plates (density of $3.2 \times 10^{4}$ cells.ml ${ }^{-1}$ ) in DMEM supplemented with 10\% FBS. Three days post seeding, the Transwells ${ }^{\circledR}$ containing differentiated hBE cells were moved to the plates containing ASM cells (marking the day 0 of the co-culture) and the co-culture was maintained in the BEDM media for 72 hours before further experiments were conducted.

\section{Alveolar epithelial and macrophage cell culture}

\section{Alveolar epithelial and alveolar macrophage cell isolation and culture}

Non-tumor lung tissue from patients undergoing lung rescission was received from the Heart Center Voelklingen. The use of human tissue was approved by the local ethic committee (State Medical Board of Registration, Saarland, Germany).

The isolation of alveolar epithelial type II cells was performed according to an established protocol [25]. Tissue cubes with an edge length of $5 \mu \mathrm{m}$ were isolated from the lung tissue and washed three times in buffer $\left(137 \mathrm{mM} \mathrm{NaCl}, 5 \mathrm{mM} \mathrm{KCl}, 0.7 \mathrm{mM} \mathrm{Na}_{2} \mathrm{HPO}_{4}, 10 \mathrm{mM}\right.$ HEPES, 5.5 mM glucose, pH 7.4) with a cell strainer (100 $\mu \mathrm{m}$ pore size; Becton Dickinson, Heidelberg, Germany). Alveolar macrophages from the first filtrate were further incubated as described below.

Lung tissue was digested in a Trypsin solution for 40 minutes at $37^{\circ} \mathrm{C}$ (Sigma Aldrich; Taufkirchen, Germany). In order to remove tissue macrophages and erythrocytes, cells were incubated on cell culture petri-dishes to for 90 minutes followed by a percoll gradient (Sigma Aldrich, Taufkirchen, Germany). The alveolar type II cells were purified with an EPCAMantibody (Miltenyi, Bergisch-Gladbach, Germany). Finally, cells were seeded on Snapwell ${ }^{\mathrm{TM}}$ - 
inserts with a pore size of $0.4 \mu \mathrm{m}$ (Corning, Schiphol-Rijk, the Netherlands), which were treated, with a mixture of fibronectin (Becton Dickinson, Heidelberg, Germany) and collagen (Sigma Aldrich, Taufkirchen, Germany). Cells were cultured in SAGM (Lonza; Verviers, Belgium) for 4 days until the co-culture was initialised.

Alveolar macrophages were washed with RPMI medium containing 5\% FCS and 1\%P/S, distributed on three cell culture petri dishes and further incubated for one hour. Cells were again washed three times with buffer and incubated in RPMI. Medium was changed after one day and the cells were cultured until day 4 (day 0 is the day of the isolation).

\section{Alveolar epithelial and alveolar macrophage co-culture}

The co-culture was prepared according to an established protocol by Hittinger et al. (manuscript in preparation [26]). Human alveolar macrophages and epithelial cells were isolated from the same donor as described above. 300,000 epithelial cells $/ \mathrm{cm}^{2}$ were seeded on a Transwell ${ }^{\mathrm{TM}}$ membrane and cultured until day 4 (day 0 is the day of isolation). Meanwhile, the macrophages were kept on petri dishes in RPMI-medium. In order to remove the macrophages from the petri dish, cells were washed 3x in PBS and incubated for 10 minutes in Trypsin/EDTA. Cells were detached with a cell scraper, washed in RPMI $1 \mathrm{x}$ and counted with a Neubauer-chamber. 100,000 alveolar macrophages/ $\mathrm{cm}^{2}$ in RPMI medium were placed on 300,000 alveolar type I-like cells on day 4 of culture. This procedure ensures that the epithelial cells form a tight barrier and macrophages are on top of the co-culture.

For air-liquid interface culture, the protocol was adjusted as macrophages do not perform strong adherence on the epithelial cells and medium cannot be simply removed from a submerged culture. In order to overcome this issue, macrophages were counted with a Neubauer-chamber 
and concentrated via centrifugation step to a small pellet. RPMI-medium was added to reach a final concentration of 100,000 macrophages in $6 \mu$ l RPMI-medium (for example: $2 \times 10^{6}$ macrophages were centrifuged, the supernatant carefully removed and $120 \mu \mathrm{l}$ RPMI medium added). $6 \mu \mathrm{l}$ of the high concentrated cell suspension were carefully dropped in the middle of each Snapwell ${ }^{\mathrm{TM}}$-insert $\left(1.12 \mathrm{~cm}^{2}\right.$ surface area) on day 4. Cells immediately spread over the whole surface and were incubated over night before performing the experiments.

In both cases, submerged and air-liquid culture conditions, the co-culture was established on day 4 and experiments were performed on day 5.

\section{Stimulation of the culture models with TGF- $\beta$}

TGF- $\beta 1$ (Invitrogen, Sydney, Australia) was added to the basal chambers at concentration of 1 $\mathrm{ng} / \mathrm{ml}$ and $10 \mathrm{ng} / \mathrm{ml}$ to stimulate the hBE cells, hAEpC and co-culture setups (hBE and ASM cells as well as hAEpC and AM, respectively). In the monoculture of hBE cells, TGF- $\beta$ was added following differentiation (21 to 28 days in culture); in the co-culture of hBE \&ASM, TGF$\beta$ was added following the third day of co-culture setup. The culture media in the basal chamber was sampled after 24 hours to measure the concentration of IL-8 using enzyme-linked immunosorbent assay (ELISA) kit (Becton Dickinson, Sydney, Australia) according to the manufacturer's protocol. Samples of the untreated mono- and co-cultures were taken as control. 


\section{Barrier integrity measurements}

Transepithelial electrical resistance was measured using a EVOM voltohmmeter (World

Precision Instruments, Sarasota, FL, USA) according to the method previously described [27].

Measurements were taken at 24 hours following stimulation with different concentrations of TGF- $\beta$ in the bronchial and alveolar mono- and co-culture systems to monitor the integrity of barrier over time following treatment with TGF- $\beta$.

TEER measurements were further confirmed by measuring the permeability of the paracellular marker fluorescein-Na (flu-Na) (Sigma-Aldrich, Sydney, Australia). The method for this study has been described elsewhere [27].

\section{Drug deposition on bronchial and alveolar epithelial cells}

\section{Deposition of SAL on hBE cells}

The Twin stage impinger (TSI) (Radleys, Essex, UK) was used to deposit SAL from Ventolin Rotacaps (GSK, Victoria, Australia) on the untreated and stimulated hBE cells grown on Transwells $^{\circledR}$. The TSI was modified, as described previously by Grainger et al., to accommodate the Transwells ${ }^{\circledR}[28]$. Following deposition of SAL, the Transwells ${ }^{\circledR}$ were transferred to a 24well plate containing $350 \mu \mathrm{l}$ of fresh pre-warmed Hank's Balanced Salt Solution (HBSS) (Gibco, Invitrogen, Sydney, Australia). At set time points (10, 30, 60, 120, 180 and 240 mins) samples were taken from the basal chamber and the amount of SAL in the samples analysed using high performance liquid chromatography. 


\section{Deposition of BD on AE cells and AM}

The pharmaceutical aerosol deposition device on cell culture (PADDOCC) was used as described previously [29] to deliver BD from Budesonide Cyclocaps (Caelo, Hilden, Germany) to the pre-treated, unstimulated and stimulated hAEpC or co-cultures of hAEpC and AM grown on Snapwells ${ }^{\mathrm{TM}}$. Six days after seeding hAEpC (24 hours following co-culture with AM), the Snapwells ${ }^{\mathrm{TM}}$ were fitted into the PADDOCC and BD microparticles were deposited on the culture of the hBE cells or the co-culture. The Snapwells ${ }^{\mathrm{TM}}$ were then transferred to a 6-well plate containing $800 \mu \mathrm{l}$ of HBSS. Samples (1, 3 and 5 hour) were assayed from the basal chamber and the amount of BD was analysed using validated high performance liquid chromatography method.

\section{Quantification of drugs in the samples}

Chemical analysis of SAL was performed with high performance liquid chromatography (HPLC), using a Shimadzu Prominence UFLC system equipped with an SPD-20A UV-Vis detector (Shimadzu Corporation, Japan) and NovaPak C18 column (5 $\mu \mathrm{m}, 150 \times 3.9 \mathrm{~mm})$ (Waters Corporation, Milford Massachusetts, USA). The mobile phase was a mixture of methanol and sodium phosphate monobasic dihydrate (30/70\%, v/v) (pH 3.2). Flow rate, injection volume and detection wavelength were $1 \mathrm{ml}^{\mathrm{min}} \mathrm{m}^{-1}, 100 \mu \mathrm{l}$ and $276 \mathrm{~nm}$, respectively. Quantification of BD was measured using UltiMate ${ }^{\circledR} 3000$ system (Dionex, Idstein, Germany) equipped with a reversed phase C18 Column (5 $\mathrm{mm}, 4.6$ × $250 \mathrm{~mm})$ (Merck KGaA, Darmstadt, Germany). The mobile phase consisted of a mixture of phosphate buffer ( $\mathrm{pH} 3$ ) and acetonitrile (60:40\%, v/v) delivered at a flow rate of $1.9 \mathrm{ml} \cdot \mathrm{min}^{-1}$. The injection volume was $100 \mu \mathrm{l}$ and BD was detected at $240 \mathrm{~nm}$. 
Measurement of pharmacological response to SAL and BD in untreated and stimulated cultures

\section{Measurement of cAMP production in culture of hBE and ASM cells}

In the co-culture model of hBE and ASM cells, following deposition of SAL using the TSI, the Transwells $^{\circledR}$ containing the hBE cells were returned to the 24-well plates containing the ASM cells. ASM cells were lysed after 4 hours of experiment and the amount of cAMP was analysed using a cAMP ELISA assay kit (Perkin Elmer, Massachusetts, USA) according to the manufacturer's protocol.

Furthermore, monoculture of ASM cells were exposed to similar concentration of SAL in the basal chamber following 4 hours of transport study and the concentration of cAMP produced was analysed and compared to the cAMP concentration produced by ASM cells in the co-culture condition.

\section{Measurement of IL-8 and IL-6 production in culture of hAEpC and AM}

In alveolar mono- and co-culture setups, TGF- $\beta$ was added to the basal chamber at the fifth day of culture setup and samples of the culture medium were taken 24 hours following stimulation

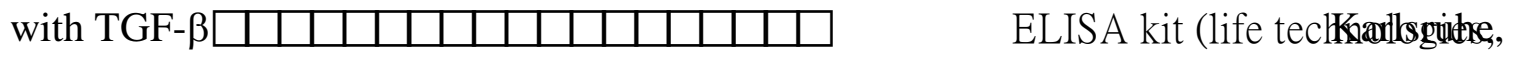
Germany). The concentrations of IL-6 and -8 in the media (basal chamber) of the unstimulated and TGF- $\beta$ stimulated cells were compared with the IL-6 and -8 concentrations of unstimulated and TGF- $\beta$ stimulated of the same cell culture setups 24 hours following deposition of BD. 
Furthermore, results were compared to IL-6 and -8 concentrations from the alveolar cells pretreated with $1 \mu \mathrm{M}$ BD on the fifth day of seeding followed by TGF- $\beta$ stimulation day 6 . The samples were taken 24 hours after stimulation to investigate the preventive anti-inflammatory role of BD in TGF- $\beta$ stimulated cells.

\section{Statistical analysis of data}

Data were analysed using GraphPad Prism 6.0 software (GraphPad Software, San Diego, USA) and presented as mean \pm standard deviation (SD). KS normality test defined the results to be normally distributed. Statistical significance was determined by paired t-test, one-way analysis of variance (ANOVA) or two-way ANOVA comparison test, where appropriate. All p values of less than 0.05 were considered significant. 


\section{Results}

\section{Effect of TGF- $\beta$ on hBE and ASM cells in vitro}

\section{Transepithelial electrical resistance}

The TEER value of the hBE cells cultured for 21-28 days at air-interface configuration was 459 $\pm 27 \Omega \cdot \mathrm{cm}^{2}$. Similar values were measured for the culture of hBE and ASM from the same patients after 3-4 days of co-culture (422 $\left.\pm 35 \Omega \cdot \mathrm{cm}^{2}\right)$. In the monoculture of hBE cells, stimulation with $10 \mathrm{ng} / \mathrm{ml}$ TGF- $\beta$ resulted in a significant increase in TEER values $(\mathrm{p} \leq 0.05)$ while a significant increase in TEER values of the co-culture $(\mathrm{p} \leq 0.05)$ was observed only when the cells were stimulated with $1 \mathrm{ng} / \mathrm{ml}$ TGF- $\beta$.

In general, the TEER values of co-cultures were slightly lower than monocultures of cells isolated from the same patients. Furthermore, there was a significant difference observed in TEER values of mono- and co- cultures following stimulation with $10 \mathrm{ng} / \mathrm{ml}$ TGF- $\beta$ with the values measured (521 $\left.\pm 27 \Omega \cdot \mathrm{cm}^{2}\right)$ and $\left(451 \pm 37 \Omega \bullet \mathrm{cm}^{2}\right)$ for mono- and co-culture, respectively ( $\mathrm{p} \leq 0.05$ ). Figure 1a shows the change in TEER following exposure to different concentrations of TGF- $\beta$ in mono- and co- cultures over 24 hours.

\section{Flu-Na paracellular permeability}

The permeability to flu-Na for both mono- and co-culture is shown in Figure 1b. For mono- and co- culture systems, permeability of the untreated cells were measured to be $(4.1 \pm 2.3) \times 10^{-6}$ $\mathrm{cm} . \mathrm{s}^{-1}$ and $(4.7 \pm 1.0) \times 10^{-6} \mathrm{~cm} . \mathrm{s}^{-1}$, respectively. No significant difference was observed between 
the permeability of the mono- and co- culture systems in control conditions and following stimulation with TGF- $\beta$ at 1 and $10 \mathrm{ng} / \mathrm{ml}$ concentrations $(p \geq 0.05)$.
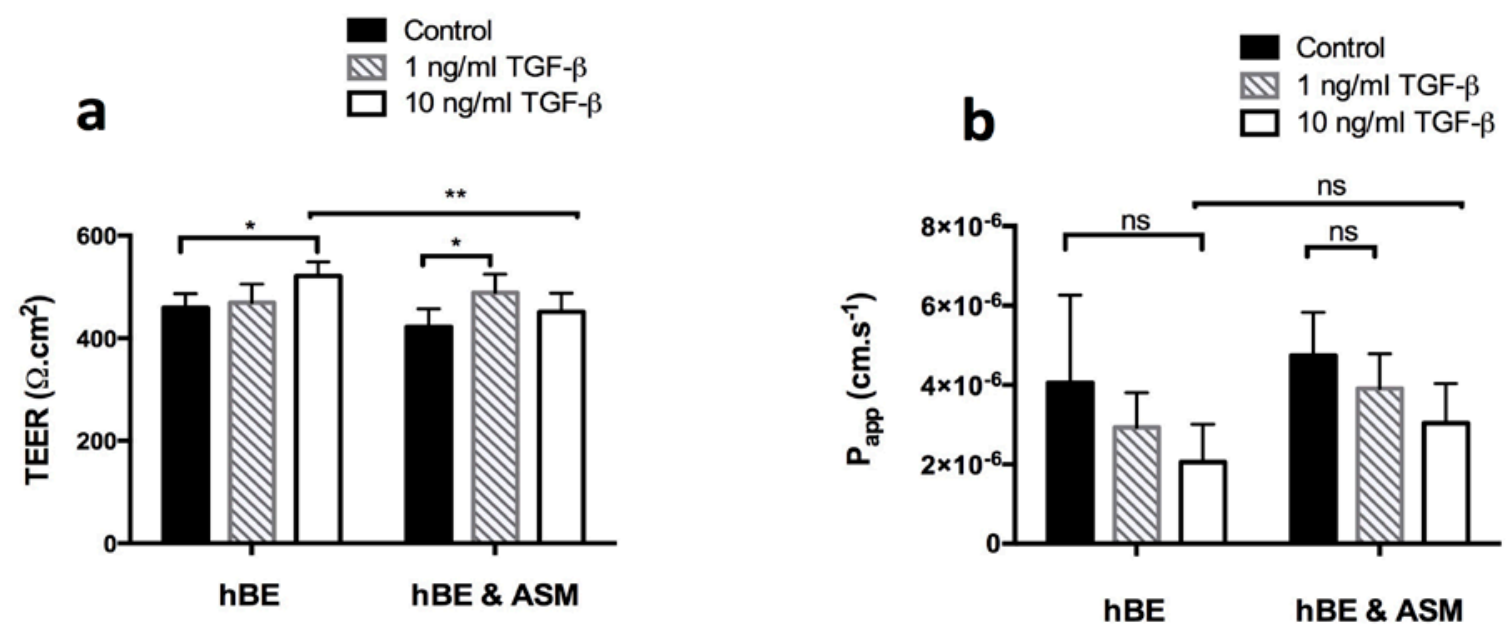

Figure 1. a) TEER and b) paracellular permeability values of the mono- and co- culture of hBE and hBE \& ASM cells to flu-Na, ( $\mathrm{n}=5, \mathrm{hBE}$ and ASM were isolated from the same patients) in the absence and presence of TGF- $\beta$ (24 hours stimulation, $1 \mathrm{ng} / \mathrm{ml}$ and $10 \mathrm{ng} / \mathrm{ml}$ ).

\section{Transport of SAL across hBE cells in mono- and co-culture setups}

Transport of SAL across hBE cells in the mono- and co-culture setting is depicted in Figure 2. In general, there was no significant difference $(\mathrm{p} \geq 0.05)$ between the average percentages of SAL transported across TGF- $\beta$ stimulated (48.47 $\pm 8.12 \%)$ vs. untreated hBE cells (54.46 \pm 15.05\%). In contrast to monoculture transport result, in the co-culture setup stimulation with TGF$\beta$ resulted in a significant difference $(\mathrm{p} \leq 0.05)$ in the average percentage of SAL transported across hBE cells with the values measured (56.53 $\pm 10.26 \%)$ and (45.12 $\pm 8.19 \%)$ for untreated

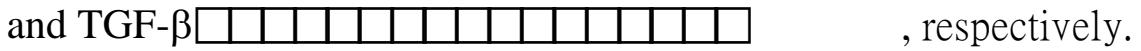


Comparison between untreated and TGF- $\beta$ stimulated cultures of individual patients cells revealed that cells from some patients responded more dramatically $(\mathrm{p} \leq 0.05)$ to stimulation with TGF- $\beta$ over 24 hours, while in other patients TGF- $\beta$ stimulation did not reduce the transport significantly ( $\mathrm{p} \geq 0.05)$.

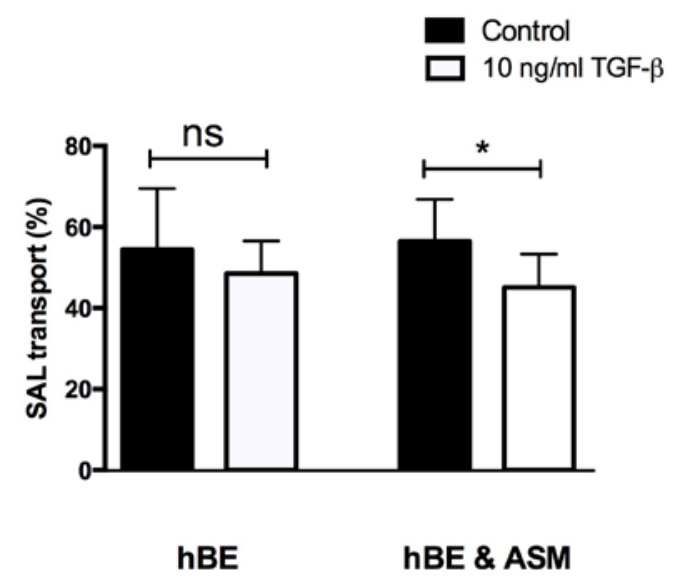

Figure 2. Epithelial transport of Salbutamol (SAL) across: A) monocultures of hBE (n=12), B) co-cultures of hBE \& ASM cells ( $\mathrm{n}=5$, hBE and ASM were isolated form the same patients) in the absence and presence of TGF- $\beta$ ( 24 hours stimulation, $10 \mathrm{ng} / \mathrm{ml}$ ).

\section{Effect of TGF- $\beta$ on IL-8 production in mono- and co- culture setups}

Following stimulation of the mono- and co-cultures with TGF- $\beta$, samples of the culture media in the basal chamber were analysed and the amount of IL-8 produced was determined by ELISA. Without such stimulation, IL-8 was not significantly different between the monoculture and the co-culture systems, with the values measured being $165 \pm 53 \mathrm{pg} / \mathrm{ml}$ and $189 \pm 58 \mathrm{pg} / \mathrm{ml}$, respectively ( $\mathrm{p} \geq 0.05)$. 
Following 10ng/ml TGF- $\beta$ stimulation, the monoculture of hBE cells showed a significant reduction of the IL-8 production (91 $\pm 30 \mathrm{pg} / \mathrm{ml})$, whereas in the co-culture model stimulation with $1 \mathrm{ng} / \mathrm{ml}$ and $10 \mathrm{ng} / \mathrm{ml}$ TGF- $\beta$ resulted in a significant increase in IL-8 secretion to $280 \pm 60$ $\mathrm{pg} / \mathrm{ml}$ and $290 \pm 31 \mathrm{pg} / \mathrm{ml}$, respectively ( $\mathrm{p}$ 0.05) (Figure 3).

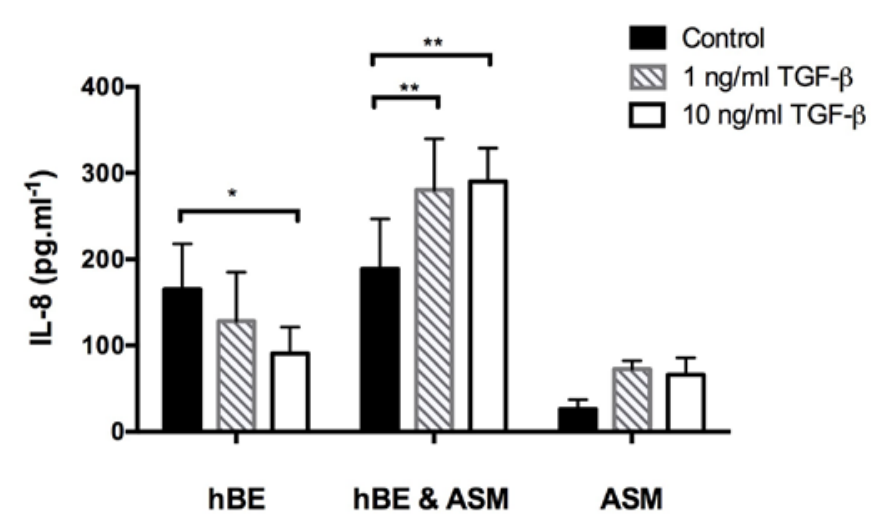

Figure 3. Changes in IL-8 expression level in cultures of hBE, hBE \& ASM and ASM cells ( $\mathrm{n}=5, \mathrm{hBE}$ and ASM were isolated form the same patients) in the absence and presence of TGF$\beta$ (24 hours stimulation, $1 \mathrm{ng} / \mathrm{ml}$ and $10 \mathrm{ng} / \mathrm{ml}$ ).

\section{Effect of TGF- $\beta$ on pharmacological responsiveness to SAL in mono- and co-culture setups}

The pharmacological response to the same concentration of SAL, either resulting after transport across hBE cells to the basolateral chamber or after direct application of the corresponding amount of SAL to the supernatant of the mono-culture of ASM cells was measured via cAMP production. The cAMP concentration was $8.1 \pm 1.0 \mathrm{nM}$ in monoculture of ASM and $7.8 \pm 1.0$ $\mathrm{nM}$ in the co-culture model. No significant difference was observed in mono- vs. co- culture condition ( $\mathrm{p} \geq 0.05$ ) (Figure 4, black bars). 
In comparison, upon 24 hours stimulation with TGF- $\beta$, the response to SAL (as reflected by the cAMP concentrations in the culture media) was significantly decreased in both culture setups to the average of $3.2 \pm 1.0 \mathrm{nM}$ in co-culture and $6.5 \pm 1.0 \mathrm{nM}$ in ASM monoculture $(\mathrm{p} \leq 0.05)$

(Figure 4, white bars), indicating the influence of TGF- $\beta$ in non-responsiveness to SAL.

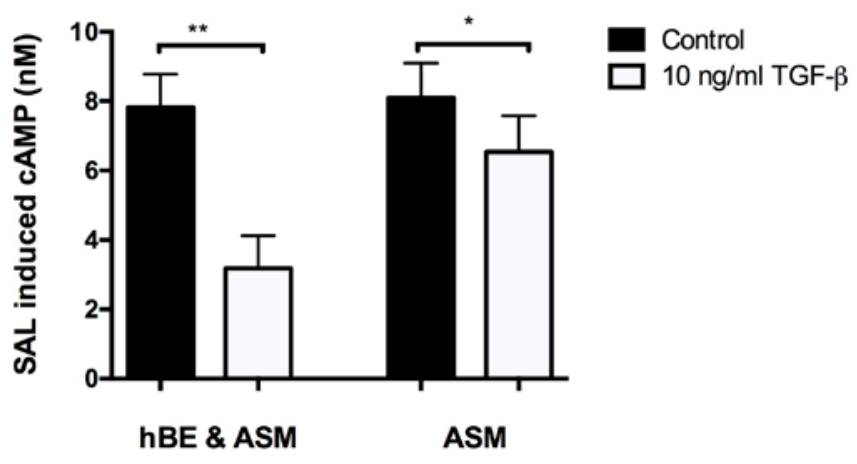

Figure 4. SAL-induced cAMP in culture of airway smooth muscle cells (mono- and co-culture setups) ( $\mathrm{n}=5$, hBE and ASM were isolated form the same patients) in the absence and presence of TGF- $\beta$ (24 hours stimulation, $10 \mathrm{ng} / \mathrm{ml}$ ).

\section{Effect of TGF- $\beta$ on hAEpC and AM in vitro}

\section{Transepithelial electrical resistance}

The TEER values measured for the monoculture of hAEpC and co-culture of hAEpC and AM at day 6 post seeding and 24 hours following co-culture are shown in Figure 5a. When comparing TEER values from all patients, there was no statistical significance when comparing mono- to co-culture ( $\mathrm{p} \geq 0.05)\left(431 \pm 30 \Omega \cdot \mathrm{cm}^{2}\right.$ for monoculture vs. $417 \pm 22 \Omega \cdot \mathrm{cm}^{2}$ for co-culture). 
Furthermore, stimulation with TGF- $\beta$ ( $1 \mathrm{ng} / \mathrm{ml}$ and $10 \mathrm{ng} / \mathrm{ml})$ for 24 hours did not significantly alter the TEER ( $p \geq 0.05$ ). Similar as previously found for hBE culture, cells obtained from the same patient showed a consistent increase in TEER value following stimulation with TGF- $\beta$ compared to the non-stimulated control regardless of mono- or co-culture conditions (data not shown).

\section{Flu-Na paracellular permeability}

In general, results from the permeability studies were in good agreement with the TEER values obtained. For cells derived from each patient, there was no significant difference in permeability of the co-culture compared to the monoculture ( $\mathrm{p} \geq 0.05)$. Furthermore, treatment of the alveolar mono- and co- culture models with $10 \mathrm{ng} / \mathrm{ml}$ TGF- $\beta$ did not result in a significant change in the permeability to flu-Na $\left((4.1 \pm 1.00) \times 10^{-6} \mathrm{~cm} \cdot \mathrm{s}^{-1}\right.$ in untreated and $\left.(3.4 \pm 0.45) \times 10^{-6} \mathrm{~cm} \cdot \mathrm{s}^{-1}\right)$ in stimulated co-cultures and $\left((3.5 \pm 0.7) \times 10^{-6} \mathrm{~cm} \cdot \mathrm{s}^{-1}\right.$ in untreated and $\left.(2.7 \pm 0.6) \times 10^{-6} \mathrm{~cm} . \mathrm{s}^{-1}\right)$ in stimulated monocultures. Figure $\mathbf{5 b}$ shows the permeability of untreated and TGF- $\beta$ stimulated hAEpC and the co-culture of hAEpC and AM.
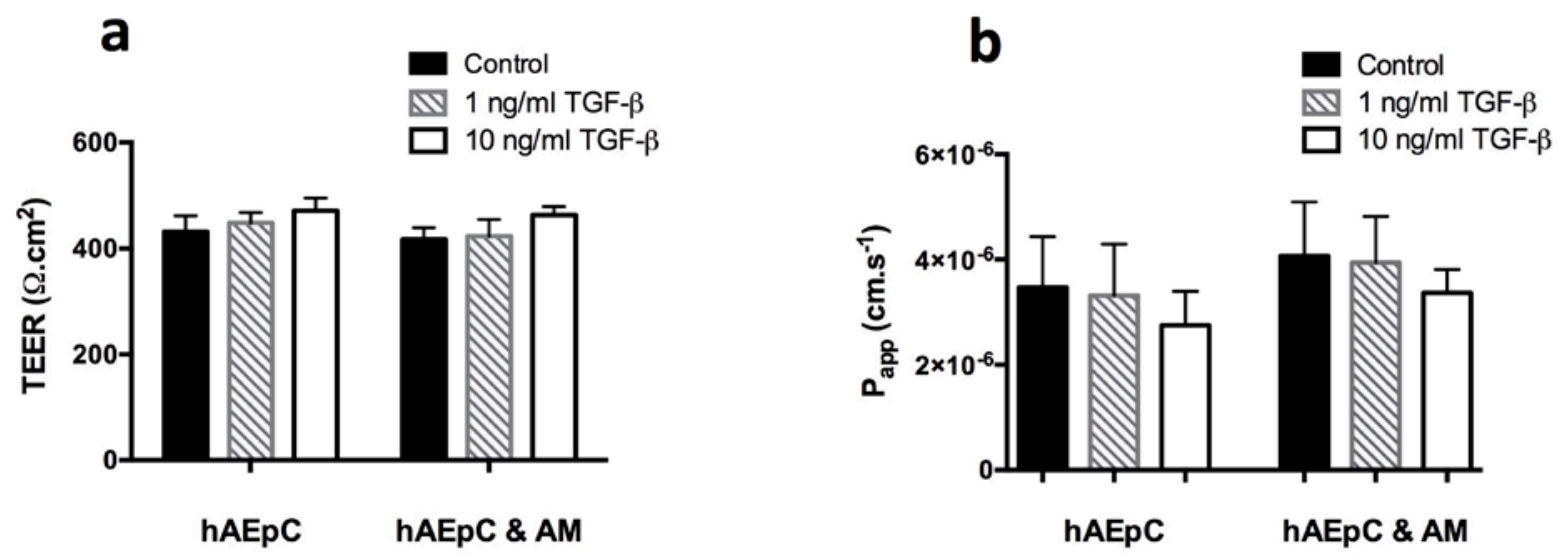
Figure 5. a) TEER and b) paracellular permeability of the mono- and co- culture of hAEpC and hAEpC \& AM to flu-Na ( $\mathrm{n}=4, \mathrm{hAEpC}$ and AM were isolated from the same patient) in the absence and presence of TGF- $\beta$ (24 hours stimulation, $1 \mathrm{ng} / \mathrm{ml}$ and $10 \mathrm{ng} / \mathrm{ml}$ ).

\section{Transport of BD across hAEpC in mono- and co- culture setups}

Figure 6 shows the percentage of BD transported following deposition on the unstimulated and stimulated mono- and co-culture setups. The results demonstrate that transport of $\mathrm{BD}$ across alveolar epithelial cells was not significantly different in mono- and co- culture systems (p $\geq$ 0.05). Furthermore, no statistically significant change was observed in the average value of BD transport across both alveolar culture setups following 24 hours treatment with TGF- $\beta$ ( $p \geq 0.05$ ).

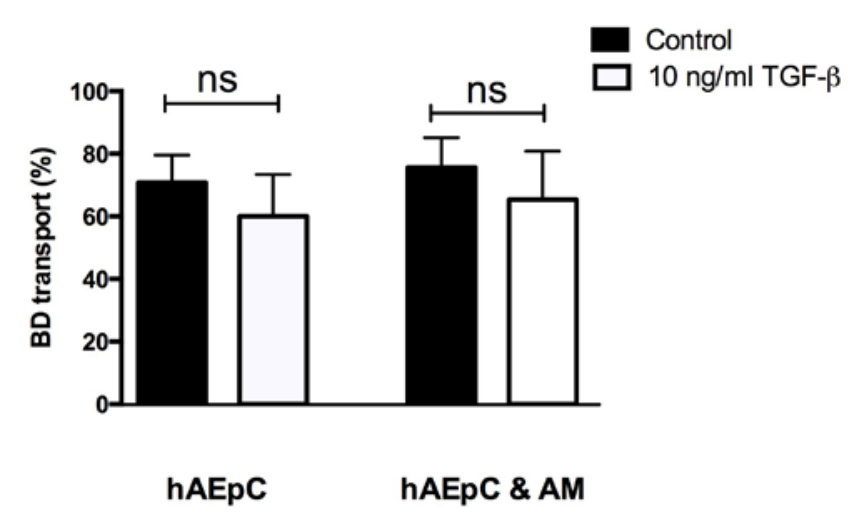

Figure 6. Epithelial transport of BD across a) monocultures of hAEpC, b) co-cultures of hAEpC \& AM ( $\mathrm{n}=4, \mathrm{hAEpC}$ and AM were isolated form the same patients) in the absence and presence of TGF- $\beta$ (24 hours stimulation, $10 \mathrm{ng} / \mathrm{ml})$. 


\section{Effect of TGF- $\beta$ on IL-6 and IL-8 production in mono- and co- culture systems}

Figure 7a and $7 \mathbf{b}$ show the concentration of IL-6 and -8 as a result of stimulation with TGF- $\beta$ and, its modulation by BD.

In the hAEpC monoculture and co-culture setup, stimulation with TGF- $\beta$ (10 ng/ml)(white bars) increased the IL-6 concentration significantly ( $p \leq 0.05$ ), compared to the IL-6 levels from unstimulated cells (black bars) (for monoculture the IL-6 level increased significantly from 186 $\pm 39 \mathrm{pg} / \mathrm{ml}$ to $528 \pm 99 \mathrm{pg} / \mathrm{ml}$; in co-culture setup the value was increased from $301 \pm 41 \mathrm{pg} / \mathrm{ml}$ to $790 \pm 120 \mathrm{pg} / \mathrm{ml}$ ). Furthermore, the IL-6 level was significantly lower in the culture of the cells (both hAEpC monoculture and co-culture) pre-treated with $1 \mu \mathrm{M}$ BD prior to stimulation (grey bars) in comparison with the stimulated cells (white bars) $(\mathrm{p} \leq 0.05)$. The significant decrease was also observed when BD was deposited 24 hours post TGF- $\beta$ stimulation ( $\mathrm{p} \leq 0.05$ ). This indicates the capability of BD in maintaining and reducing the IL-6 concentration at control level.

Furthermore, the difference observed in IL-6 levels produced in hAEpC monoculture and the coculture setup following stimulation was also significant ( $\mathrm{p} \leq 0.05$ ). In the monoculture of AM alone, the changes in IL-6 levels were not statistically significant following stimulation, pretreatment and $\mathrm{BD}$ treatment of the AMs and IL-6 levels were measured to be significantly lower in the AM monoculture in comparison with the co-culture model following stimulation and BD treatment $(\mathrm{p} \leq 0.05)$.

In the monoculture of hAEpC, the significant change was only observed between the IL-8 levels in cells stimulated with TGF- $\beta$ (white bars) $(129 \pm 21 \mathrm{pg} / \mathrm{ml})$ and the cells pre-treated with $1 \mu \mathrm{M}$ 
$\mathrm{BD}$ (grey bars) $(84 \pm 18 \mathrm{pg} / \mathrm{ml})(\mathrm{p} \leq 0.05)$. In the co-culture setup, however, IL-8 level from the cells stimulated with TGF- $\beta$ (white bar) $(127 \pm 23 \mathrm{pg} / \mathrm{ml})$ was significantly lower than the unstimulated cells (black bar) $(195 \pm 25 \mathrm{pg} / \mathrm{ml})(\mathrm{p} \leq 0.05)$ and was measured significantly higher than the cultures pre-treated with $1 \mu \mathrm{M}$ BD (grey bar) $(65 \pm 9 \mathrm{pg} / \mathrm{ml})(\mathrm{p} \leq 0.05)$ and treatment with BD following 24 hours of stimulation did not impact the amount of IL-8 expressed ( $\mathrm{p} \geq$ 0.05). In the monoculture of AM, a significant reduction was observed in IL-8 levels in the cultures pre-treated with BD (grey bars) and the IL-8 levels in unstimulated (black bars) and TGF- $\beta$ stimulated cells ( $\mathrm{p} \leq 0.05)$.
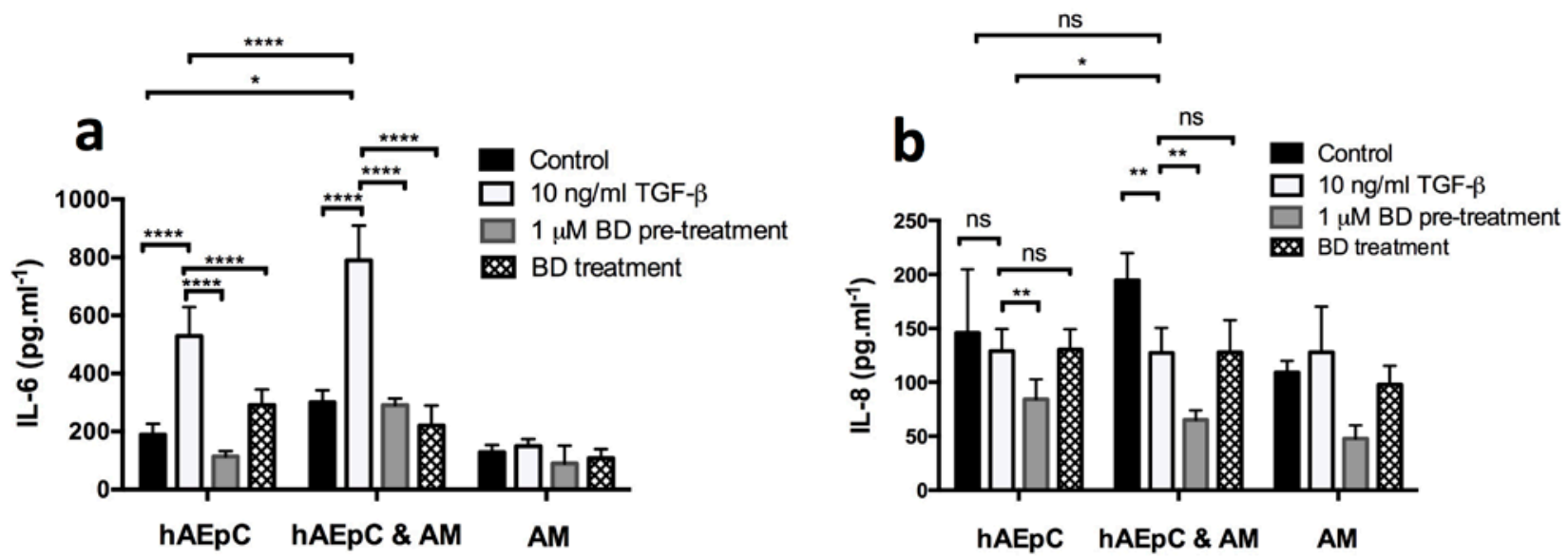

Figure 7. Changes in a) IL-6 and b) IL-8 expression level in cultures of hAEpC and hAEpC \& AM in the absence of TGF- $\beta$ (black bars), following 24 hours stimulation with TGF- $\beta$ (10 ng/ml) (white bars), pre-treatment with $1 \mu \mathrm{M}$ BD prior to stimulation with TGF- $\beta$ (grey bars)

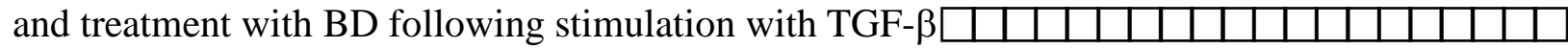
( $\mathrm{n}=4, \mathrm{hAEpC}$ and AM were isolated form the same patients). 


\section{Discussion}

This is the first study to compare mono- and co- culture models of primary bronchial (cocultured with ASM) and alveolar epithelial cell (co-cultured with AM) models as barrier to drug transport while simultaneously investigating the therapeutic effectiveness of the drugs following stimulation with TGF- $\beta$.

Our results demonstrated that the permeability and TEER values of the untreated cells for both bronchial and alveolar mono- and co- culture models were similar, and no significant difference was observed in the measurements. In the bronchial cells cultures, stimulation with TGF- $\beta$ over 24 hours resulted in a significant increase in TEER, while no significant change was observed in paracellular permeability. This finding indicates that while TEER and paracellular permeability of the air-interface cultured epithelial cells from bronchial and alveolar regions of the lung are not significantly different, they respond differently to TGF- $\beta$ stimulation.

The average transport of SAL across untreated hBE cells in mono- and co-culture settings was not significantly different. However, the percentage of SAL transported varied between 38 to $78 \%$ of the deposited dose in epithelial cultures obtained from different patients. A more detailed analysis of individual patients' cells revealed that the differences observed were not relevant to the background disease, age, sex or smoking history of the patients but rather pointing to the importance of inter-individual differences. Furthermore, stimulation with TGF- $\beta$ in bronchial coculture setup resulted in an overall significant decrease of SAL transport. Similarly, in the alveolar mono- and co- culture models, transport of BD across untreated cells was not significantly different and the percentage of transport was measured to be between 65 to $90 \%$ of 
the deposited dose of the drug. Stimulation with $10 \mathrm{ng} / \mathrm{ml}$ TGF- $\beta$ did not significantly impact BD transport across the hAEpC in mono- or co-culture setup.

It was observed that in unstimulated and TGF- $\beta$ stimulated bronchial and alveolar mono- and cocultures, transport of SAL and BD ranged between 38 to $78 \%$ and 65 to $90 \%$, respectively. One possible explanation could be the inter-individual difference in the membrane transporters expressed on the epithelial cells. It has been shown that transport of SAL is mediated via passive diffusion as well as the transporter proteins (i.e. organic cationic transporters-OCTs) [21, 30], while $\mathrm{BD}$ being lipophilic is transported by passive diffusion [31]. Furthermore, BD is known to be a substrate of P-glycoprotein [32], an efflux transporter that limits BD absorption by pumping it back into the airways. Therefore, the inter-individual differences in P-glycoprotein could have contributed to the differences in BD absorption. Interestingly, the inter-individual differences are also observed following stimulation with TGF- $\beta$ in the bronchial and alveolar cultures and the percentage of drug transported was affected more dramatically in some patient's cells.

Furthermore, the results demonstrated that TGF- $\beta$ stimulation of the bronchial co-culture resulted in a significant decrease in SAL transport. In a previous study by Unwalla et al. [33] that reported the paracellular route was the major contributor to SAL flux across the airway epithelium. While a decrease in SAL transport following stimulation confirms the previous study, there was no evidence of a significant decrease in paracellular permeability or significant increase in TEER. This could be attributed to the fact that the mentioned study was conducted using tracheal epithelial cells from two subjects, while in the current study bronchial cells from more subjects were studied, which provided a superior representation of the biological variability. 
$\beta_{2}$-adrenergic receptor $\left(\beta_{2}\right.$-AR) agonists such as SAL are important for symptomatic management of airway obstruction and their effectiveness is affected by their transport across the epithelial barrier to get to the $\beta_{2}$-AR on ASM cells. Reduced cAMP production and reduction in the number of $\beta_{2}$-AR were reported to be the result of reduced responsiveness of ASM cells following treatment with TGF- $\beta$ [34]. In this study a reduced cAMP release upon treatment of TGF- $\beta$ stimulated cells by SAL was observed in the hBE-ASM and ASM cultures, although the decrease was more dramatic in the hBE \& ASM co-cultures. In addition, in the present study the inter-individual differences observed in transport of SAL could have contributed to the cAMP levels observed.

Corticosteroids such as BD are the most effective therapy targeting airway inflammation in asthma and COPD [1, 2], However, COPD is a disease characterised by steroid insensitivity, and some patients with asthma, especially those with severe disease are also refractory to steroid treatment [35]. Reduction in the transport of BD in some patients following stimulation with TGF- $\beta$, as observed in present study, could result in the interindividual differences in the amount of BD transported.

In a previous study by Ge et al., [36] it was demonstrated that stimulation of hBE cells with TGF- $\beta 1$ (1 ng/ml) resulted in a significant decrease in IL-8 levels. While in the present study the decrease in IL-8 levels measured in monoculture of hBE, hAEpC and co-culture of hAEpC \& AM are in agreement with Ge et al., in the bronchial co-culture model (hBE \& ASM), TGF- $\beta$ caused a significant increase in IL-8 levels.

Cell culture models of bronchial and respiratory epithelium have been developed to provide a platform for studying drug transport and uptake. While the longer established 2D monocultures 
of epithelial cells are more suitable for high throughput screening, easier to use and less costly, they are on the other hand more reductionist and therefore less suitable to perform mechanistic studies or to investigate pathophysiological processes. Due to their reduced complexity monocultures are less suitable to monitor changes in epithelial barrier properties as a result of respiratory diseases and do not take into account the role of other local cell types in the fate and therapeutic effectiveness of drugs. Novel advanced 3D models of the pulmonary epithelia that also comprise other cell types have recently been described [36, 37], reflecting the necessary cellular crosstalk when addressing questions of toxicity, transport or inflammatory responses. Thereby, the use of complex co-culture systems compared to monocultures has a clear influence on the study outcome. Kasper et al [37] demonstrated that a pulmonary epithelial-endothelial coculture was less responsive to silica nanoparticles in terms of toxicity, while inflammatory mediators (IL-8) were increased in the co-culture setup. In case of bronchial and alveolar models of hBE and hAEpC in the current study, unstimulated mono- and co-cultures did not show significantly different responses in terms of transport and cytokine secretion, while the bronchial stimulated co-culture was able to reflect the non-responsiveness to SAL and the alveolar simulated cultures showed a significant reduction in inflammatory cytokine levels following BD treatment. This finding indicates the requirement for a pathophysiological stimulus when investigating the efficacy of therapeutic drugs.

The present study highlights the differences of monocultures vs. more complex co-cultures of different cell types and the importance of the cellular network, when biological responses (e.g. IL-8) and therapeutic effectiveness (e.g. cAMP) are studied. 


\section{Conclusion}

Taken together, the data from this study identifies that there might be intrinsic differences in the transport of SAL and BD in different patients that could manifest as clinically different responses to the drugs. Furthermore, the results of this study demonstrated the advantage of the co-cultures over monocultures and highlighted the impact of cellular interplay on the biological responses and therapeutic effectiveness of drug molecules. 


\section{Funding Sources}

This work was financially supported by the Alexander von Humboldt Foundation and the

German Federal Ministry of Education and Research (support code: 13N11458).

\section{Notes}

The authors declare no competing financial interest.

\section{References}

1. From the Global Strategy for Asthma Management and Prevention, G.I.f.A.G.A.f.h.w.g.o.

2. $\quad$ From the Global Strategy for the Diagnosis, M.a.P.o.C., Global Initiative for Chronic Obstructive Lung Disease (GOLD) 2014. Available from: http://www.goldcopd.org/.

3. Barnes, P.J., Mediators of Chronic Obstructive Pulmonary Disease. Pharmacological Reviews, 2004. 56(4): p. 515-548.

4. $\quad$ Anderson, G.P., Current issues with beta2-adrenoceptor agonists: pharmacology and molecular and cellular mechanisms. Clin Rev Allergy Immunol, 2006. 31(2-3): p. 11930.

5. $\quad$ van der Velden, V.H., Glucocorticoids: mechanisms of action and anti-inflammatory potential in asthma. Mediators of Inflammation, 1998. 7(4): p. 229-237.

6. Makinde, T., R.F. Murphy, and D.K. Agrawal, The regulatory role of TGF-[beta] in airway remodeling in asthma. Immunol Cell Biol, 2007. 85(5): p. 348-356.

7. Wang, R.D., J.L. Wright, and A. Churg, Transforming Growth Factor- $\beta 1$ Drives Airway Remodeling in Cigarette Smoke-Exposed Tracheal Explants. American Journal of Respiratory Cell and Molecular Biology, 2005. 33(4): p. 387-393.

8. Barnes, P.J., The cytokine network in asthma and chronic obstructive pulmonary disease. The Journal of Clinical Investigation, 2008. 118(11): p. 3546-3556.

9. Takizawa, H., et al., Increased Expression of Transforming Growth Factor- $\beta 1$ in Small Airway Epithelium from Tobacco Smokers and Patients with Chronic Obstructive Pulmonary Disease (COPD). American Journal of Respiratory and Critical Care Medicine, 2001. 163(6): p. 1476-1483.

10. McKay, S. and H. Sharma, Autocrine regulation of asthmatic airway inflammation: role of airway smooth muscle. Respiratory Research, 2002. 3(1): p. 11. 
11. Barnes, P.J. and J.M. Drazen, Chapter 35 - Pathophysiology of Asthma, in Asthma and COPD, P.J.B.M.D.R.C. Thomson, Editor. 2002, Academic Press: London. p. 343-359.

12. Lotvall, J., in Advances in Combination Therapy for Asthma and COPD, J. Lotvall, Editor. 2011, Wiley-Blackwell.

13. Barnes, P.J., Glucocorticosteroids: current and future directions. Br J Pharmacol, 2011. 163(1): p. 29-43.

14. Adcock, I.M. and P.J. Barnes, Molecular mechanisms of corticosteroid resistance. Chest, 2008. 134(2): p. 394-401.

15. Pelaia, G., et al., Molecular mechanisms of corticosteroid actions in chronic inflammatory airway diseases. Life Sci, 2003. 72(14): p. 1549-61.

16. Irusen, E., et al., p38 Mitogen-activated protein kinase-induced glucocorticoid receptor phosphorylation reduces its activity: role in steroid-insensitive asthma. J Allergy Clin Immunol, 2002. 109(4): p. 649-57.

17. Morishima, Y., et al., Th17-Associated Cytokines as a Therapeutic Target for SteroidInsensitive Asthma. Clinical and Developmental Immunology, 2013. 2013: p. 9.

18. Ito, K., et al., Histone deacetylase 2-mediated deacetylation of the glucocorticoid receptor enables NF-kappaB suppression. J Exp Med, 2006. 203(1): p. 7-13.

19. Barnes, P.J., K. Ito, and I.M. Adcock, Corticosteroid resistance in chronic obstructive pulmonary disease: inactivation of histone deacetylase. Lancet, 2004. 363(9410): p. 7313.

20. Hittinger, M., et al., Preclinical safety and efficacy models for pulmonary drug delivery of antimicrobials with focus on in vitro models. Advanced Drug Delivery Reviews, (0).

21. Haghi, M., et al., Deposition, diffusion and transport mechanism of dry powder microparticulate salbutamol, at the respiratory epithelia. Mol Pharm, 2012. 9(6): p. 1717-26.

22. Haghi, M., D. Traini, and P. Young, In vitro cell integrated impactor deposition methodology for the study of aerodynamically relevant size fractions from commercial pressurised metered dose inhalers. Pharm Res, 2014. 31(7): p. 1779-87.

23. Haghi, M., et al., Fluticasone uptake across Calu-3 cells is mediated by salmeterol when deposited as a combination powder inhaler. Respirology, 2013. 18(8): p. 1197-201.

24. Van Ly, D., et al., Characterising the mechanism of airway smooth muscle beta2 adrenoceptor desensitization by rhinovirus infected bronchial epithelial cells. PLoS One, 2013. 8(2): p. e56058.

25. Daum, N., et al., Isolation, Cultivation, and Application of Human Alveolar Epithelial Cells, in Human Cell Culture Protocols, R.R. Mitry and R.D. Hughes, Editors. 2012, Humana Press. p. 31-42.

26. Hittinger, M., et al., Autologous co-culture of human alveolar cells for safety testing of airborne particles. ALTEX, 2015.

27. Haghi, M., et al., Time- and passage-dependent characteristics of a Calu-3 respiratory epithelial cell model. Drug Dev Ind Pharm, 2010. 36(10): p. 1207-14.

28. Grainger, C.I., et al., The permeability of large molecular weight solutes following particle delivery to air-interfaced cells that model the respiratory mucosa. Eur J Pharm Biopharm, 2009. 71(2): p. 318-24.

29. Hein, S., et al., A new Pharmaceutical Aerosol Deposition Device on Cell Cultures (PADDOCC) to evaluate pulmonary drug absorption for metered dose dry powder formulations. Eur J Pharm Biopharm, 2011. 77(1): p. 132-8. 
30. Horvath, G., et al., Epithelial organic cation transporters ensure pH-dependent drug absorption in the airway. Am J Respir Cell Mol Biol, 2007. 36(1): p. 53-60.

31. Borchard, G., et al., Transport and local metabolism of budesonide and fluticasone propionate in a human bronchial epithelial cell line (Calu-3). J Pharm Sci, 2002. 91(6): p. 1561-7.

32. Dilger, K., M. Schwab, and M.F. Fromm, Identification of budesonide and prednisone as substrates of the intestinal drug efflux pump P-glycoprotein. Inflamm Bowel Dis, 2004. 10(5): p. 578-83.

33. Unwalla, H.J., et al., Albuterol Modulates Its Own Transepithelial Flux via Changes in Paracellular Permeability. American Journal of Respiratory Cell and Molecular Biology, 2012. 46(4): p. 551-558.

34. Mak, J.C.W., et al., Transforming growth factor- $\beta 1$ inhibits $\beta 2$-adrenoceptor gene transcription. Naunyn-Schmiedeberg's Archives of Pharmacology, 2000. 362(6): p. 520525.

35. Barnes, P.J., Corticosteroid Resistance in Airway Disease. Proceedings of the American Thoracic Society, 2004. 1(3): p. 264-268.

36. Ge, Q., et al., TGFbeta1 induces $I L-6$ and inhibits $I L-8$ release in human bronchial epithelial cells: the role of Smad2/3. J Cell Physiol, 2010. 225(3): p. 846-54. 